\title{
Optimizing periodicity and polymodality in noise-induced genetic oscillators
}

\author{
Pau Rué, ${ }^{1}$ Gürol M. Süel, ${ }^{2}$ and Jordi Garcia-Ojalvo ${ }^{1, *}$ \\ ${ }^{1}$ Departament de Física i Enginyeria Nuclear, Universitat Politècnica de Catalunya, Edifici GAIA, \\ Rambla de Sant Nebridi s/n, Terrassa, E-08222 Barcelona, Spain \\ ${ }^{2}$ Green Center for Systems Biology and Department of Pharmacology, University of Texas Southwestern Medical Center, \\ Dallas, Texas 75390, USA
}

(Received 6 January 2011; revised manuscript received 22 March 2011; published 6 June 2011)

\begin{abstract}
Many cellular functions are based on the rhythmic organization of biological processes into self-repeating cascades of events. Some of these periodic processes, such as the cell cycles of several species, exhibit conspicuous irregularities in the form of period skippings, which lead to polymodal distributions of cycle lengths. A recently proposed mechanism that accounts for this quantized behavior is the stabilization of a Hopf-unstable state by molecular noise. Here we investigate the effect of varying noise in a model system, namely an excitable activator-repressor genetic circuit, that displays this noise-induced stabilization effect. Our results show that an optimal noise level enhances the regularity (coherence) of the cycles, in a form of coherence resonance. Similar noise levels also optimize the multimodal nature of the cycle lengths. Together, these results illustrate how molecular noise within a minimal gene regulatory motif confers robust generation of polymodal patterns of periodicity.
\end{abstract}

DOI: 10.1103/PhysRevE.83.061904

PACS number(s): 87.18.Tt, 87.18.Vf

\section{INTRODUCTION}

Biological oscillations underlie many physiological functions in cells, from basic processes such as cell growth and division [1] to evolutionary environmental adaptations such as circadian rhythmicity [2]. Many circuit architectures have been proposed that explain the observed periodic behavior in terms of limit-cycle attractors of nonlinear dynamical models [3]. These limit cycles exhibit a perfectly periodic behavior, which is only slightly perturbed by realistic levels of biochemical random fluctuations, or noise, that are unavoidable in cells [4]. In some situations, however, cellular oscillations display a degree of variability much larger than what can be obtained from a limit-cycle model with added noise. This is the case, for instance, of the cell cycle oscillations exhibited by Chinese hamster cells [5], fission yeast cells [6], and Xenopus laevis blastomeres [7]. In these organisms, cells do not always divide when they are supposed to, giving rise to a distribution of cell-cycle periods that is not unimodal, but that exhibits secondary peaks at multiples of the cell-cycle period. This quantized behavior cannot be explained by the usual factors responsible for the heterogeneity of the cell cycle, such as parameter inhomogeneities and the age distribution of cells within a population. Those factors, which are undoubtedly present in any dividing cell population, would only lead to broadening of the period distribution but not to polymodality. Therefore detailed mathematical models with a relatively large number of biochemical components (on the order of 10) have been proposed to explain this behavior [8]. In those models, period skipping arises already at the deterministic level (i.e., in the absence of sources of heterogeneity and inhomogeneity) [9], while noise is sometimes considered [10] to reproduce the level of irregularity observed in the experiments. Other striking examples of polymodal cycles

*jordi.g.ojalvo@upc.edu embedded in an otherwise oscillatory dynamics were reported long ago in sensory neurons [11] and bacterial motility [12].

Using the phenomenologies described above as motivation, here we address the general question of how a limit cycle behavior with polymodal period distribution can arise in minimal oscillator models. To that end we consider one of the most basic oscillator architectures, namely a two-component activatorinhibitor system operating in an excitable regime (close to the oscillatory region) and subject to noise. We recently showed that such a model system exhibits noise-induced stabilization of an unstable spiral state [13]. Due to its excitable character, this model system displays noise-triggered excursions away from the stable (rest) state, during which the cell passes through a region near the unstable spiral. The stabilization mechanism consists in the appearance of oscillations around the unstable state, due to the stochastic fluctuations. As a consequence of these oscillations, the distribution of excursion times away from the rest state exhibits a marked polymodality: each noiseinduced oscillation around the unstable state introduces a well defined delay (the oscillation period) in the pulse duration. Thus noise can explain the polymodality of pulse duration distributions in certain conditions. However, the (excitable) pulses are triggered by noise to begin with, and thus they are far from occurring periodically, which would be necessary if this mechanism is to explain the polymodal cell-cycle duration distributions mentioned above.

Coincidentally, however, systems with excitable dynamics are known to exhibit enhanced periodicity, or coherence, for an optimal amount of noise: too little noise will elicit pulses only sparsely, and therefore irregularly, while too high noise will lead to a strong disorder in the dynamics. A moderate level of noise, on the other hand, is able to evoke pulses frequently, as soon as the refractory time following the previous pulse (characteristic of all excitable systems) has elapsed, and thus leads to a substantially periodic behavior, with a period basically given by the refractory time. Such somewhat counterintuitive effect of noise has been termed coherence resonance or, more 
appropriately, stochastic coherence [14-16]. The goal of this paper is to show that stochastic coherence can be invoked, together with the noise-induced stabilization effect discussed above, to provide a minimal mechanism for the generation of polymodal distributions of cycle lengths in an otherwise periodic behavior. The mechanism requires only a simple genetic activator-repressor motif and an optimal amount of random fluctuations. In our setting, the effect of intrinsic molecular noise is characterized by using discrete stochastic simulations.

The level of intrinsic noise is controlled by the cell volume, whose increase (together with the gene copy numbers) effectively scales up the numbers of molecular species (thus reducing the noise), while maintaining the concentrations constant. This approach was recently introduced experimentally in B. subtilis [17], and has been subsequently used in E. coli as well [18]. From the theoretical side, system-size effects have been seen to lead to stochastic-resonance $[19,20]$ and stochastic-coherence [21] effect through their control of the effective noise intensity perceived by the system.

Our results show that noise, besides enhancing the regularity of the pulse activations, also optimizes polymodality in the system's response. Furthermore, optimization of periodicity and polymodality are achieved when noise levels are comparable. Thus when the coherence of the excitable pulses is maximized, so is the probability that the pulses undergo oscillations around the unstable spiral state. There is a range of noise levels for which optimization holds. Together, these results show that noisy activator-repressor genetic circuits can naturally behave as polymodal oscillators.

\section{MODEL}

We now describe our model system using the terminology of gene regulation circuits, although the results obtained are applicable to any activator-inhibitor system. The genetic circuit (see Fig. 1) is a simple two-component system where an activator protein, $A$, binds to and activates its own promoter, $P_{a}$, and the promoter of a repressor species $P_{r}$. The repressor component, $R$, in turn, inhibits the expression of the activator species by competitively binding to the promoter $P_{a}$. This system is a prototypical transcriptional activator-repressor genetic circuit where the activator species forms a direct positive feedback loop through its autoregulation and an indirect negative feedback loop by means of the activation of its own repressor [22-24]. The dynamics of this system is highly nonlinear, due to the cooperative nature of the regulated transcription processes. Specifically, the kinetics of the regulated activation of the $P_{a}$ promoter is described by a Hill function with cooperativity $n$ :

$$
f\left(A, R, k_{2}, K_{a}, K_{i}, n, m\right)=\frac{k_{2} A^{n}}{A^{n}+K_{\mathrm{eff}}^{n}},
$$

where $A$ represents the activator concentration in the cell, and the effective activation threshold $K_{\text {eff }}$ depends on the concentration $R$ of repressor through the expression $K_{\text {eff }}^{n}=$ $K_{a}^{n}\left[1+\left(R / K_{i}\right)^{m}\right]$. In this equation, the term $K_{i}$ accounts for the competitive inhibition exerted by $R$, the net effect of which is to increase the promoter's activation threshold. The kinetics (a)

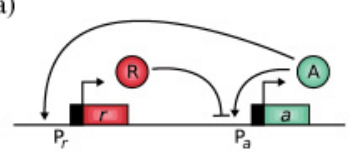

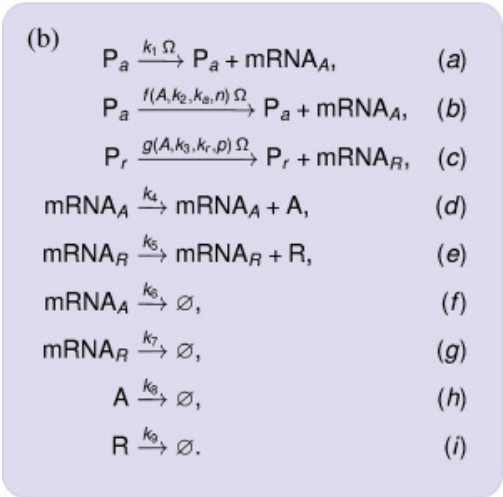

FIG. 1. (Color online) (a) The genetic motif investigated here consists of two genes, $a$ and $r$, coding for activator $A$ and a repressor $R$ proteins, respectively. The activator protein binds to the promoters $P_{a}$ and $P_{r}$ of the genes $a$ and $r$, respectively, and the repressor protein binds to $P_{a}$ competitively inhibiting its activation by $A$. (b) Set of reactions describing the genetic circuit. Reaction (a) corresponds to the leaky or constitutive transcription of activator mRNA. Regulated transcription of the activator and repressor mRNA species is encoded in reactions (b) and (c). Protein translation for the activator and repressor is represented by reactions (d) and (e), respectively, and degradation reactions for the mRNA and protein species are (f)-(i).

of regulated transcription of the repressor mRNA is described by a simple activation Hill equation,

$$
g\left(A, k_{3}, K_{r}, p\right)=\frac{k_{3}}{1+\left(\frac{K_{r}}{A}\right)^{p}},
$$

with constant activation threshold $K_{r}$.

The values of the reaction rates used in the simulation shown below are given in Table I. The values of the parameters are within reasonable biological ranges for a gene regulation circuit. In particular, the values chosen for the transcription, translation, and degradation rates, and for the activation and inhibition threshold concentrations, are of the same order of magnitudes of previous studies that involved qualitative comparison and careful validation with experimental measurements $[17,25,26]$. Note that the rates of the transcription reactions in Fig. 1 are proportional to a factor $\Omega$.

TABLE I. Values of the reaction rates used in the stochastic simulations of the circuit represented in Fig. 1.

\begin{tabular}{lcll}
\hline \hline$k_{1}$ & $0.00625 \mathrm{nM} /(\mathrm{s}$ molecule $)$ & $k_{6}$ & $0.05 \mathrm{~s}^{-1}$ \\
$k_{2}$ & $0.5 \mathrm{nM} /(\mathrm{s}$ molecule $)$ & $k_{7}$ & $0.05 \mathrm{~s}^{-1}$ \\
$k_{3}$ & $0.05 \mathrm{nM} /(\mathrm{s}$ molecule $)$ & $k_{8}$ & $0.001 \mathrm{~s}^{-1}$ \\
$k_{4}$ & $2 \mathrm{~s}^{-1}$ & $k_{9}$ & $0.0001 \mathrm{~s}^{-1}$ \\
$k_{5}$ & $2 \mathrm{~s}^{-1}$ & $K_{a}$ & $5000 \mathrm{nM}$ \\
$K_{r}$ & $9000 \mathrm{nM}$ & $K_{i}$ & $5000 \mathrm{nM}$ \\
$n$ & 2 & $m$ & 2 \\
$p$ & 4 & & \\
\hline
\end{tabular}


This parameter is a global scaling factor that depends on the size of the cell. Specifically, $\Omega=V \cdot N_{A}$, where $V$ is the cell volume and $N_{A}$ is Avogadro's number. In that way, $\Omega$ relates the species concentrations with the number of molecules: if $n_{A}$ is the number of molecules of $A$ and $A$ is its concentration, then $n_{A}=\Omega A$. We will also consider that the transcription strengths are proportional to $\Omega$, which is a generalization to the continuum of the assumption that the gene copy number increases with cell size. This happens when a cell is prevented from dividing but not from replicating its DNA, as happens in certain bacterial mutants [17]. Under those assumptions, the level of molecular noise increases monotonically with the inverse of the system size $\Omega^{-1}$ [27]. In the following, we will use the parameter $\Omega^{-1}$ to characterize the levels of noise in the system.

A continuous model of the circuit for the case of negligible fluctuations can be derived for the set of reactions listed in Fig. 1(b) [28]. Let $a, r$ denote the concentration of activator and repressor mRNA molecules and $A, R$ the protein concentrations. Applying standard kinetics rules to the reactions listed in Fig. 1(b) leads in a straightforward way to the following coupled ordinary differential equations:

$$
\begin{gathered}
\frac{d a}{d t}=k_{1}+k_{2} \frac{A^{n}}{A^{n}+K_{a}{ }^{n}+\left(\gamma_{r} R\right)^{m}}-k_{6} a, \\
\frac{d r}{d t}=k_{3} \frac{A^{m}}{K_{r}{ }^{p}+A^{p}}-k_{7} r, \\
\frac{d A}{d t}=k_{4} a-k_{8} A, \\
\frac{d R}{d t}=k_{5} r-k_{9} R .
\end{gathered}
$$

where $\gamma_{r}{ }^{m}=K_{a}{ }^{n} / K_{i}{ }^{m}$. Note that this system of equations is independent of the system size.

Further assuming a separation of mRNA and protein time scales, the former ones (being much smaller) can be adiabatically eliminated, and the system can be reduced to two coupled ordinary differential equations,

$$
\begin{gathered}
\frac{d A}{d t}=\alpha+\beta_{1} \frac{A^{n}}{A^{n}+K_{a}^{n}+\left(\gamma_{r} R\right)^{m}}-\lambda_{1} A, \\
\frac{d R}{d t}=\beta_{2} \frac{A^{p}}{K_{r}{ }^{p}+A^{p}}-\lambda_{2} R,
\end{gathered}
$$

where

$$
\begin{gathered}
\alpha=k_{1} k_{4} / k_{6}, \\
\beta_{1}=k_{2} k_{4} / k_{6}, \quad \beta_{2}=k_{3} k_{5} / k_{7}, \\
\lambda_{1}=k_{8}, \quad \lambda_{2}=k_{9} .
\end{gathered}
$$

While in this work the model dynamics is obtained by exact discrete stochastic simulation of the chemical kinetics [27] described in Fig. 1(b), its qualitative dynamical aspects are described with the planar system of differential equations (2). We note that, as in the case of Eqs. (1), our final deterministic model given by Eqs. (2) does not depend on the system size, and thus the deterministic dynamics will be unchanged as noise levels vary. This is due to the fact that the system size factor $\Omega$ rescales the levels of all proteins (and thus the absolute activation and inhibition thresholds) in the same manner as the transcription rates, while keeping the concentrations unchanged. In that way, the average concentration dynamics of the model does not depend on noise, but only the variances of the concentrations do.

\section{RESULTS}

\section{A. Deterministic excitable dynamics and effects of molecular noise}

The interplay between the autoactivation positive feedback loop of $A$ on itself and the negative feedback loop formed by the activator and the repressor allow for a wide range of rich dynamics. In particular, for the set of parameters given in Table I and Eq. (3) the system is excitable. The phase portrait depicting the nullclines of this system for those parameter values is shown in Fig. 2(a). The system has three equilibrium points: a stable node, a saddle point, and an unstable focus. In the absence of noise, the system rests in the only stable state, which in this case corresponds to low numbers of both activator and repressor molecules. Small perturbations from this stable
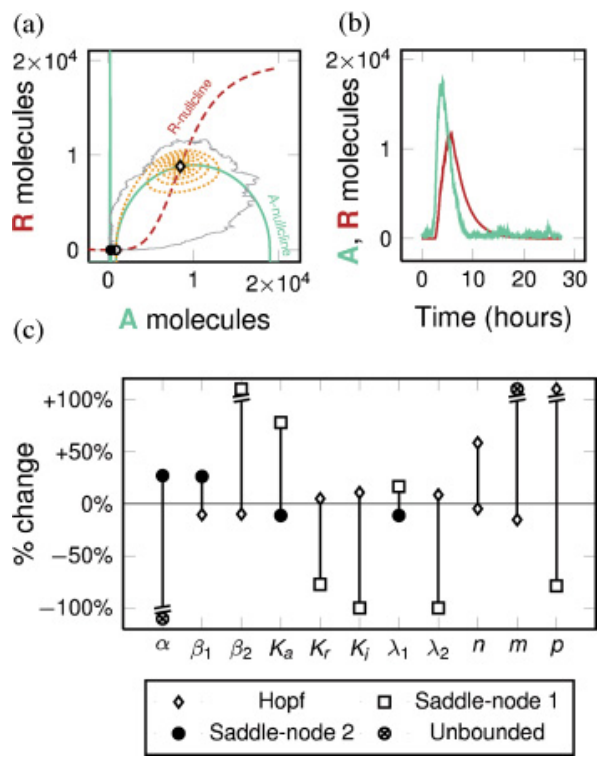

FIG. 2. (Color online) (a) Phase portrait representing the dynamics of the system of equations (2). The nullclines for $A$ and $R$ are represented by solid (green online) and dashed (red online) lines. The system has three equilibrium points: a stable node (black circle), a saddle point (white circle), and an unstable focus (white diamond). The stable manifold of the saddle point (dotted line, yellow online) introduces a threshold of excitability. A trajectory obtained by discrete stochastic simulations $(\Omega=1$ molecule $/ \mathrm{nM})$ is shown in black. The random fluctuations of this system make it possible to cross the separatrix and initiate a large excursion in the phase plane. (b) The same trajectory is plotted as a time course of the protein species. When the threshold of excitability is crossed, a transient pulse in the number of activator and repressor molecules is produced. (c) Sensitivity of the model to single parameter changes. Vertical lines show the range of parameter values for which excitability is maintained (in \% change from the values in Table I). The symbols at the end of the vertical lines indicate the type of bifurcation leading to loss of excitability, or whether the range is unbounded (see legend and main text). Broken bars indicate much larger ranges than the one indicated by the vertical axis. 
point vanish exponentially and the system quickly recovers the rest state. However, the stable manifold of the saddle point [the separatrix, dotted spiral line in Fig. 2(a)] is an excitability threshold beyond which perturbations evoke a large excursion through phase space, passing around the unstable focus and back to the stable point avoiding crossing the separatrix [29]. The occurrence of this excitability cycle can be understood as follows: a sufficiently large initial amount of activator molecules triggers the positive feedback loop and leads to a large pulse of activator molecules. The increasing levels of activator switch on the production of repressor proteins (repressor pulse) which, in turn, shut down the production of activator. Finally, the amounts of activator and repressor decay due to linear degradation or dilution. This transient response is characterized by a refractory time, which is the duration of the cycle from the triggering event to the recovery of the rest state.

Stochastic fluctuations due to intrinsic noise can destabilize the rest state by randomly crossing the excitability threshold and hence generating pulses of activator and repressor protein levels. Figure 2(a) shows an excursion in phase space triggered by stochastic fluctuations, and Fig. 2(b) shows the corresponding time course for both the activator and the repressor.

The excitable regime in which this system operates arises when the system is close to a bifurcation point beyond which the dynamics has the form of a limit cycle [30]. In addition, the noise-induced stabilization of the unstable state emerges close to a Hopf bifurcation [13] beyond which the system becomes bistable. Despite these constraints in the parameter values, the activator-repressor system presented here is robust to parameter changes, as shown in Fig. 2(c). This figure depicts the main bifurcations from excitability that the system undergoes as the kinetic parameters are varied one by one. In particular, the codimension-1 bifurcations found are (i) the stabilization of the unstable spiral via a Hopf bifurcation; (ii) the collision of the saddle and the node defining the resting state [saddle node 1 in Fig. 2(c)]; and (iii) the collision of the saddle and the unstable state (saddle node 2). This analysis shows that the more sensitive parameters are the activator degradation rate $\lambda_{1}$, for which the excitability is maintained for a global range of variation of $27.8 \%$, and the the maximum regulated transcription rate $\beta_{1}$, for which a global variation of $36.7 \%$ is possible without losing excitability.

\section{B. Polymodality in the cycle duration depends on the level of intrinsic noise}

As already mentioned, the sporadic generation of pulses of activity is not the only effect caused by molecular noise in this system. As shown in Figs. 3(a) and 3(b), noise also stabilizes the unstable state and generates bursts, or cycles, of multiple pulses. This is due to the fact that noise causes the trajectories traveling around the unstable spiral point to cross over the stable manifold of the saddle [dotted line in Fig. 2(a)]. This leads to the trajectory getting trapped orbiting around the unstable fixed point for an integer number of cycles, thus generating a polymodal distribution of pulse durations [13].

In order to characterize this noise-induced polymodality, we have computed the cycle durations for varying levels of molecular noise (which increase as the system size $\Omega$
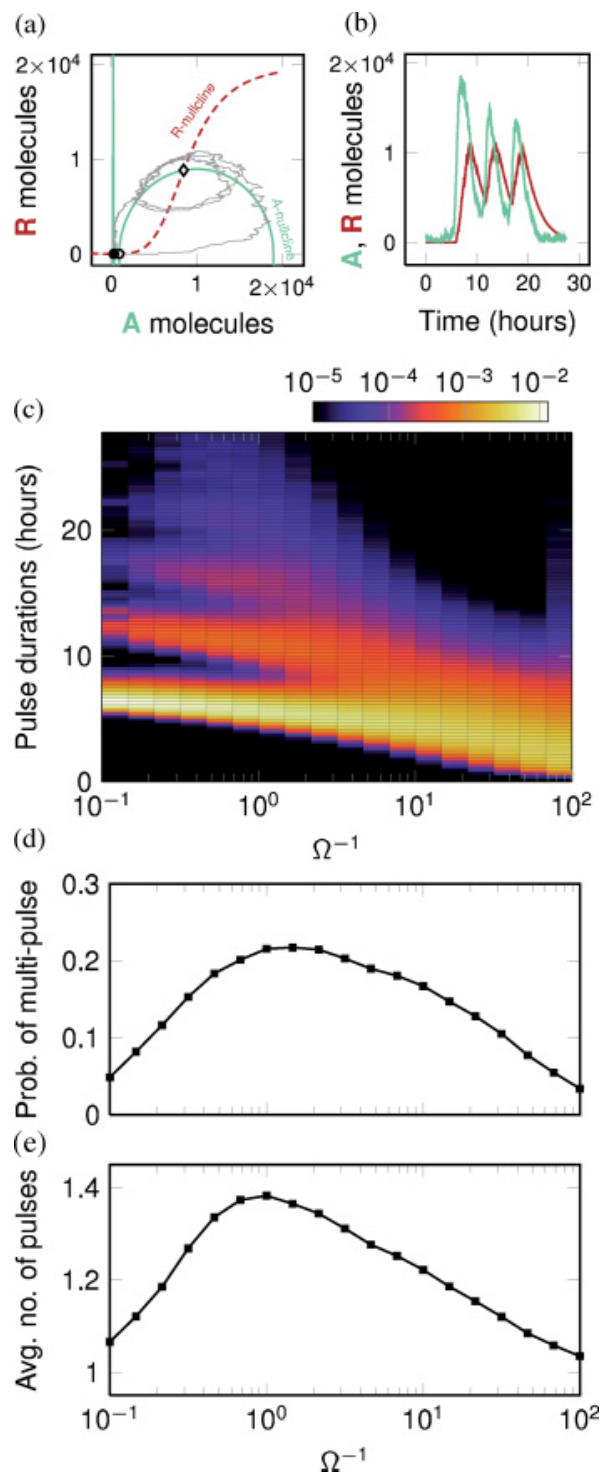

FIG. 3. (Color online) Molecular noise induces bursts with multiple pulses. (a) A burst trajectory obtained with discrete stochastic simulations $(\Omega=1 \mathrm{molecule} / \mathrm{nM})$ is depicted in the phase plane. Fluctuations due to molecular noise synergistically interact with the dynamics around the focus and can temporarily trap the system in an area around the otherwise unstable state. (b) Time course for the number of activator and repressor proteins for the burst shown in (a). The stabilization of the active state is characterized by the oscillations in the numbers of molecules. (c) Histograms of the burst durations for varying levels of the intrinsic noise $\left(\Omega^{-1}\right)$. Color is coded in logarithmic scale to emphasize the existence of polymodality. (d) Probability of generating a cycle with multiple pulses plotted against the noise level. For intermediate values of the noise strength the system reaches a maximum probability of generating bursts with more than one pulse. (e) Average number of pulses per burst of activity as a function of $\Omega^{-1}$.

decreases). Figure 3(c) shows the normalized histogram of burst durations for different values of $\Omega^{-1}$. For large system sizes (small $\Omega^{-1}$, small molecular fluctuations), the histogram shows multiple modes with clearly defined peaks. Each of the modes of the histogram obviously corresponds to a class of burst with a definite number of pulses. The single pulse 
cycle (corresponding to the first mode in the histogram) is, by far, the most probable case. For intermediate noise levels, the secondary peaks, corresponding to cycles with multiple pulses, get both higher and wider. If noise is increased further, the polymodal character of cycle durations is lost and just a single wide peak remains.

These results insinuate a new resonance-like effect in which growing levels of noise increase the polymodality in the pulse-duration distribution, until it reaches a maximum and starts declining again. In order to assess this resonant effect, we have computed the probability of having more than one pulse in one burst [Fig. 3(d)], together with the number of pulses per activation burst [Fig. 3(e)] as a function of increasing noise levels. Here, the pulses per cycle are computed by counting the number of complete cycles around the unstable focus. This method was found to be a very robust way to compute the number of pulses in the presence of large random fluctuations. Both plots clearly show the predicted resonance effect, with the optimal degree of polymodality arising at a value of $\Omega \sim 1$ molecule $/ \mathrm{nM}$.

The reasoning behind this resonant effect can be stated as follows. For large system sizes (small noise), noise is large enough to trigger excitable pulses, but it is too small to easily induce crossings of the trajectory beyond the stable manifold of the saddle. Thus the fraction of bursts in which there is more than one cycle is small (and the average number of cycles is close to 1). On the opposite side, for small system sizes, the random fluctuations are too large to maintain the coherence of the oscillations around the unstable spiral, and the burst duration is no longer quantized, but is widely variable and with small mean, since it is easy for the trajectory to escape the area near the unstable spiral and relax back to the neighborhood of the rest state. For intermediate system sizes, on the other hand, noise is large enough to induce frequent crossings of the spiral's stable manifold, but small enough to maintain the coherence of the noise-induced oscillations, and thus polymodality is maximized.

\section{Noise modulates the regularity of the oscillations}

Let us now turn our attention to the ability of the system to generate regular cycles. Not being a genuine genetic oscillator but an excitable system, pulses in this circuit are in principle randomly generated by noise. In this scenario, we want to establish whether the level of noise has an impact in the regularity of pulse initiation. This effect is already made evident by visual inspection of the time traces of the activator species for different values of $\Omega$, as shown in Fig. 4(a). The three panels in this figure display time traces of the the activator species for three different levels of molecular noise (increasing noise from top to bottom). The dynamics of the system in these three panels are qualitatively different. While the top and bottom panels show bursts of activity at very irregular time intervals, the middle panel presents a quite regular pattern of cycles. This plot already hints at a second noise-dependent resonancelike effect, according to which the regularity of excitable pulses is maximized for an intermediate amount of noise, which is known as coherence resonance or stochastic coherence $[15,16]$. In order to quantify this effect, we compute the coefficient of variation $(\mathrm{CV})$ of the time between bursts of
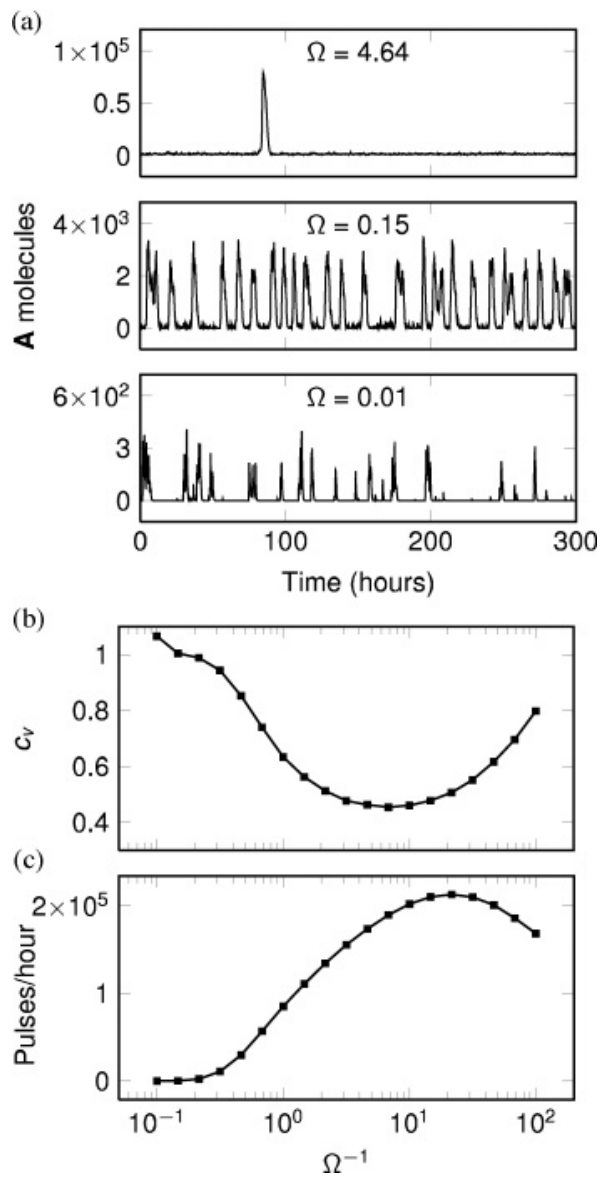

FIG. 4. (a) Temporal evolution of number of activator molecules for three different values of the system size. Note how the peak number of molecules decreases as the system size decreases. (b) Coefficient of variation of the intervals between consecutive cycle initiations, plotted versus $\Omega^{-1}$. (c) The average rate of cycle initiation as a function of the noise level. For large system sizes, the fluctuations are not large enough to initiate the cycles. For small system sizes the decay in the pulsing rate is mainly due to the discrete nature of the chemical reactions and the small amount of mRNA species.

protein concentration, a reliable regularity measure. The $\mathrm{CV}$ is defined as the standard deviation normalized to the mean,

$$
c_{v}=\frac{\sqrt{\left\langle T^{2}\right\rangle-\langle T\rangle^{2}}}{\langle T\rangle},
$$

where the random variable $T$ denotes the duration between bursts of activity. This measure is routinely used as a quantifier of noise-induced regularity in coherence-resonance studies [16]. In a perfectly periodic regime where the pulses were equally spaced in time, the coefficient of variation would be exactly zero. Conversely, in a completely random regime with pulses following poissonian statistics (exponential waiting times), the coefficient would take the value of 1 (the standard deviation being equal to the mean) or even higher (hyperexponential waiting times). Thus the smaller the value of the $\mathrm{CV}$, the closer the system is to operating in a coherent regime. Other quantifiers of coherence resonance, such as the correlation time of the dynamics, can be used, but lead to the 
same conclusions [15]. Here, the CV is estimated from the simulated time courses using a robust thresholding method.

The coherence resonance effect is revealed in Fig. 4(b), where the $\mathrm{CV}$ is plotted against the system size. For small amounts of intrinsic noise the system mainly remains in the basal stable state, with some sporadic pulses appearing randomly in time [see also Fig. 4(c), which shows that the pulsing rate approaches 0 for small noise]. This results in a $\mathrm{CV}$ of around 1. As the system size is decreased, the effect of intrinsic noise increases and the system pulses at a higher pace. Here is where the refractory time enters the game, as it poses a limit in the pulsation frequency (the system cannot undergo a new cycle if it has already started one). Thus temporal regularity appears as a synergistic effect involving the dynamics of the system and the intrinsic noise. In particular, the maximum regularity in the oscillations (a minimum CV) appears at $\Omega=0.147$ molecule $/ n M$. Further reducing the system size causes a reduction in the regularity of the oscillations. This loss of coherence is due to two main causes: first, large amounts of noise destroy the excursion paths thus generating a variety of incomplete pulses and eliminating the system's eigenfrequency dictated by the refractory time [16]. The second cause of coherence loss, which is not common in standard coherence resonance, is the appearance of periods of silencing where the activity of the system is completely shut down [see bottom panel in Fig. 4(a)]. These periods of silencing are due to the fact that when the cell size is very small, the number of molecular species is very small. In particular, the number of mRNA molecules falls frequently to zero, resulting in the total absence of protein expression during relatively large time intervals. Such low levels of mRNA are not uncommon in cells, as has been recently shown experimentally in E. coli [31].

\section{Polymodality affects regularity}

We have shown that this circuit displays two apparently opposing effects caused by intrinsic noise. On one side, noise increases the variability in the duration of the cycles in a quantized manner. On the other side, it reduces the variability in the cycle initiation times. Thus it is reasonable to think that these effects might affect one another. Here we show how polymodality in the duration of the cycle poses a limit in the temporal coherence attained by the genetic oscillator. For this purpose, we first assume that, for a given range of noise, we reach a perfect timing of the cycles, where each cycle follows the next without delay. In this hypothetical case we can estimate the loss of temporal coherence (in the $\mathrm{CV}$ sense) attributable to the cycle duration polymodality by taking into account the probabilities of obtaining a cycle with a particular number of bursts. In this case, in which a new cycle starts just after the previous one, the average time between cycles is $\left\langle T_{\mathrm{pm}}\right\rangle=\sum_{i \geqslant 1} p_{i} T_{i}$, where $p_{i}$ is the probability of getting a burst with exactly $i$ pulses $(i>0)$ and $T_{i}$ is the average duration of a burst with $i$ pulses. We can also estimate the variance by further assuming that all the cycles with $i$ pulses have a length of exactly $T_{i}$ (zero variance among them): $\sigma_{\mathrm{pm}}{ }^{2}=\left\langle T_{\mathrm{pm}}{ }^{2}\right\rangle-\left\langle T_{\mathrm{pm}}\right\rangle^{2}=\sum_{i \geqslant 1}\left(p_{i} T_{i}{ }^{2}\right)-\left\langle T_{\mathrm{pm}}\right\rangle^{2}$. This allows us to calculate the contribution of the polymodality, $c_{v}^{\mathrm{pm}}$, to the total coefficient of variation of the time between initiations.

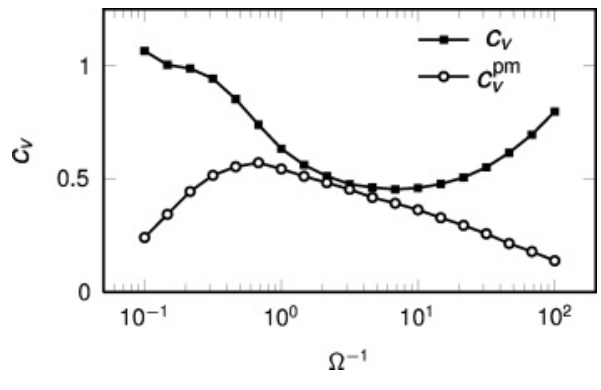

FIG. 5. Coefficient of variation of the interburst times due to polymodality $c_{v}^{\mathrm{pm}}$ (white circles), compared to the total $\mathrm{CV} c_{v}$ of the dynamics [black squares, from Fig. 4(b)]. For intermediate values of noise the $\mathrm{CV}$ of the oscillator becomes close to $c_{v}^{\mathrm{pm}}$.

Figure 5 compares $c_{v}^{\mathrm{pm}}$ (white circles) with the total CV [black squares; see also Fig. 4(b)] for varying noise levels. In this figure, $c_{v}^{\mathrm{pm}}$ has been computed using values for the probabilities $p_{i}$ that were estimated from the time traces of the stochastic simulations [see Fig. 3(d) and accompanying text]. In addition, the values for $T_{i}$ have been fitted to the formula $T_{i} / T_{1}=1+(i-1) \delta$ (resulting in $\delta=0.75)$. A comparison between the white circles in Fig. 5 and the result of Fig. 3(e) shows that, for continuous pulsing (noise generates a pulse as soon as the refractory time from the previous pulse is over), the regularity of the pulses drops ( $c_{v}^{\mathrm{pm}}$ increases), as the polymodality is enhanced. Thus in the regime of continuous pulsing there is an evident tradeoff between cycle length polymodality and temporal coherence. Finally, Fig. 5 also shows that around the minimum CV the system is close to the regime of constant cycling. Thus in that case basically all the remaining irregularity (note that the CV does not decay to zero) is due to the polymodality. Therefore polymodal behavior establishes an upper bound for the regularity of the system's dynamics.

\section{E. Noise-induced polymodality and regularity robustly coexist}

An important question is how the noise levels that optimize coherence and polymodality compare to one another. A comparison between Figs. 3(d) and 4(b) reveals that, for the parameter values chosen, there is one order of magnitude difference between the two optimal noise levels. However, we can still say that the two effects displayed by this simple genetic model take place for overlapping ranges of molecular noise. This can be argued from Fig. 6, which plots together the measures of polymodality (in the $x$ axis) and regularity (in the $y$ axis) for varying amounts of noise (milestones labeled in some data points in the figure). The figure shows that as noise increases, both the regularity and polymodality increase, and there is a range of noise levels spanning over an order of magnitude (black squares in the figure), for which both magnitudes are equally high, before decreasing again as noise is further increased. Thus one can say that an optimal level of noise maximizes almost simultaneously, and for a relatively wide range of noise amplitudes, both the regularity and the polymodality of the dynamics of the activator-repressor module. 


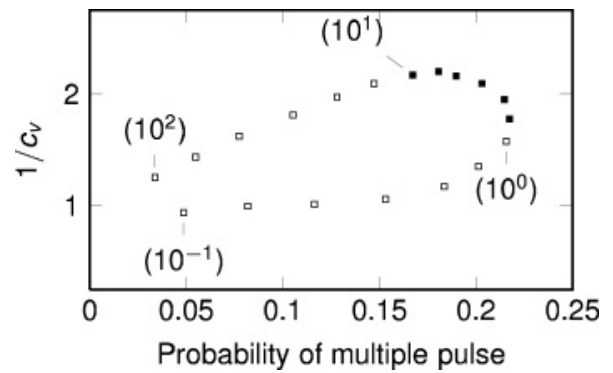

FIG. 6. A range of noise levels optimize regularity and polymodality almost simultaneously. Regularity $\left(1 / c_{v}\right.$, from Fig. 4$)$ is plotted against polymodality [probability of multiple pulses in a burst, from Fig. 3(d)] for varying levels of molecular noise. Labels indicate the inverse of the system sizes $\left(\Omega^{-1}\right)$ for some of the points. Black squares correspond to noise levels for which the system is both largely polymodal (probability $\geqslant 0.15)$ and regular $\left(c_{v}<0.6\right)$.

\section{DISCUSSION}

Cellular processes regulated by genetic components are subject to large amounts of random fluctuations. In the face of this fact, it is appealing to conjecture that, rather than simply trying to filter out noise, certain cellular mechanisms have evolved to cope with random fluctuations, and in some cases even rely on them for function. In the last decades many noise-induced phenomena in physical systems have been described, both theoretically and experimentally. Strikingly, noise can in some cases increase order in the dynamics [32] and play a constructive role in nonlinear systems. It is also becoming evident in recent years that molecular noise has an impact on the dynamics underlying many biological processes [26,33-36].

Here we have shown that intrinsic noise is able to turn a simple activator-repressor genetic circuit into an oscillator with nontrivial statistical properties, reflected in a polymodal distribution of cycle durations embedded in a relatively strongly periodic sequence. A similar effect has been reported in coupled excitable elements $[37,38]$. Here, in contrast, we show that the phenomenon can arise in single excitable elements. The role of noise in our system is twofold. On the one hand, it stabilizes an unstable spiral point by inducing oscillations around it, which increases the duration of phasespace excursions in a quantized manner. Furthermore, the resulting polymodal character of the dynamics is enhanced for an intermediate noise level. The second role of noise is to enhance coherence in the pulse initiation times, which occurs via a standard coherence resonance effect, characteristic of excitable systems subject to noise [16]. This double optimization provides a relatively simple mechanism for the emergence of polymodal behavior in genetic oscillators.

\section{ACKNOWLEDGMENTS}

We thank Ekkehard Ullner for useful discussions. This work has been financially supported by the Ministerio de Ciencia e Innovación (Spain, Project No. FIS2009-13360). P.R. was supported by the FI program from the Generalitat de Catalunya. G.M.S. acknowledges support by NIH Grant No. NIGMS RO1 GM088428, Welch Foundation (Grant No. I-1674), and James S. McDonnell Foundation (Grant No. 220020141). J.G.O. also acknowledges financial support from the ICREA Foundation.
[1] J. J. Tyson, K. Chen, and B. Novak, Nat. Rev. Mol. Cell Biol. 2, 908 (2001).

[2] A. Goldbeter, Nature (London) 420, 238 (2002).

[3] B. Novák and J. J. Tyson, Nat. Rev. Mol. Cell Biol. 9, 981 (2008).

[4] D. Gonze, J. Halloy, and A. Goldbeter, Proc. Natl. Acad. Sci. USA 99, 673 (2002).

[5] R. R. Klevecz, Proc. Natl. Acad. Sci. USA 73, 4012 (1976).

[6] A. Sveiczer, B. Novak, and J. M. Mitchison, J. Cell Sci. 109, 2947 (1996).

[7] Y. Masui and P. Wang, Biol. Cell 90, 537 (1998).

[8] A. Sveiczer, A. Csikasz-Nagy, B. Gyorffy, J. J. Tyson, and B. Novak, Proc. Natl. Acad. Sci. USA 97, 7865 (2000).

[9] B. Novak, Z. Pataki, A. Ciliberto, and J. J. Tyson, Chaos 11, 277 (2001).

[10] R. Steuer, J. Theor. Biol. 228, 293 (2004).

[11] J. E. Rose, J. F. Brugge, D. J. Anderson, and J. E. Hind, J. Neurophysiol. 30, 769 (1967).

[12] A. Schimz and E. Hildebrand, Proc. Natl. Acad. Sci. USA 89, 457 (1992).

[13] M. Turcotte, J. Garcia-Ojalvo, and G. M. Süel, Proc. Natl. Acad. Sci. USA 105, 15732 (2008).

[14] Hu Gang, T. Ditzinger, C. Z. Ning, and H. Haken, Phys. Rev. Lett. 71, 807 (1993).
[15] A. S. Pikovsky and J. Kurths, Phys. Rev. Lett. 78, 775 (1997).

[16] B. Lindner, J. García-Ojalvo, A. Neiman, and L. SchimanskyGeier, Phys. Rep. 392, 321 (2004).

[17] G. M. Suel, R. P. Kulkarni, J. Dworkin, J. Garcia-Ojalvo, and M. B. Elowitz, Science 315, 1716 (2007).

[18] E. Fischer-Friedrich, G. Meacci, J. Lutkenhaus, H. Chaté, and K. Kruse, Proc. Natl. Acad. Sci. USA 107, 6134 (2010).

[19] A. Pikovsky, A. Zaikin, and M. A. de la Casa, Phys. Rev. Lett. 88, 050601 (2002).

[20] G. Schmid, I. Goychuk, and P. Hänggi, Europhys. Lett. 56, 22 (2001).

[21] R. Toral, C. R. Mirasso, and J. D. Gunton, Europhys. Lett. 61, 162 (2003).

[22] T. Y.-C. Tsai, Y. S. Choi, W. Ma, J. R. Pomerening, C. Tang, and J. E. Ferrell, Science 321, 126 (2008).

[23] J. Stricker, S. Cookson, M. R. Bennett, W. H. Mather, L. S. Tsimring, and J. Hasty, Nature (London) 456, 516 (2008).

[24] M. Tigges, T. T. Marquez-Lago, J. Stelling, and M. Fussenegger, Nature (London) 457, 309 (2009).

[25] G. Süel, J. Garcia-Ojalvo, L. Liberman, and M. Elowitz, Nature (London) 440, 545 (2006).

[26] T. Çăgatay, M. Turcotte, M. B. Elowitz, J. Garcia-Ojalvo, and G. Süel, Cell 139, 512 (2009). 
[27] D. T. Gillespie, J. Phys. Chem. 81, 2340 (1977).

[28] U. Alon, An Introduction to Systems Biology (Chapman and Hall/CRC, London, 2006).

[29] E. M. Izhikevich, Dynamical Systems in Neuroscience: The Geometry of Excitability and Bursting (MIT, Cambridge, MA, 2006).

[30] P. Rué and J. Garcia-Ojalvo, Math. Biosci. 231, 90 (2011).

[31] Y. Taniguchi, P. J. Choi, G.-W. Li, H. Chen, M. Babu, J. Hearn, A. Emili, and X. S. Xie, Science 329, 533 (2010).

[32] F. Sagués, J. M. Sancho, and J. García-Ojalvo, Rev. Mod. Phys. 79, 829 (2007).
[33] A. J. McKane and T. J. Newman, Phys. Rev. Lett. 94, 218102 (2005).

[34] D. Alonso, A. J. McKane, and M. Pascual, J. R. Soc. Interface 4, 575 (2007).

[35] A. Eldar and M. B. Elowitz, Nature (London) 467, 167 (2010).

[36] M. Kittisopikul and G. M. Süel, Proc. Natl. Acad. Sci. USA 107, 13300 (2010).

[37] E. I. Volkov, M. N. Stolyarov, A. A. Zaikin, and J. Kurths, Phys. Rev. E 67, 66202 (2003).

[38] A. Koseska, E. Volkov, A. Zaikin, and J. Kurths, Phys. Rev. E 76, 020901 (2007). 